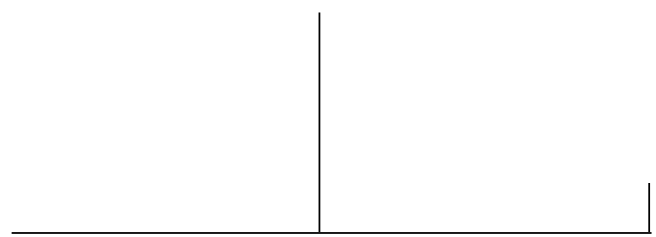

Rev. Latinoam. Psicopat. Fund., São Paulo, v. 15, n. 4, p. 891-907, dezembro 2012

\title{
La noción de inconsciente en Freud: antecedentes históricos y elaboraciones teóricas*
}

Miguel Gallegos

\begin{abstract}
Este trabajo aborda la noción de inconsciente desde una perspectiva histórica. La noción de inconsciente es una de las categorías centrales del psicoanálisis y reconoce diversos antecedentes teóricos. En primer lugar, se expone el origen del término en la reflexión filosófica y en las referencias de la psiquiatría. En segundo lugar, se trabaja el inconciente en las elaboraciones teóricas de Freud. Por ultimo, se exponen las diversas formas en las que Freud legitima la noción de inconsciente en el marco del psicoanálisis.
\end{abstract}

Palabras clave: Inconsciente, Freud, historia, psicoanálisis

\footnotetext{
*Este trabajo se realizó gracias a una beca de posgrado del Consejo Nacional de Investigaciones Científicas y Técnicas (Conicet), de Argentina.
} 


\title{
Introducción
}

\begin{abstract}
Hacía mucho tiempo que el concepto de inconciente golpeaba a las puertas de la psicología para ser admitido. Filosofía y literatura jugaron con él harto a menudo, pero la ciencia no sabía emplearlo. El psicoanálisis se ha apoderado de este concepto, lo ha tomado en serio, lo ha llenado con un contenido nuevo.
\end{abstract}

(Freud, 1938, p. 288).

El concepto de inconsciente si bien puede rastrearse en el tiempo más remoto de la historia del pensamiento humano, no obstante, es una categoría que ha cobrado una significación más precisa a partir del desarrollo de la teoría psicoanalítica propuesta por Sigmund Freud a principios de siglo XX. La noción de inconsciente se ha ido incorporando al cuerpo de conceptos usados socioculturalmente y se la reconoce como una categoría sinónimo de Freud y de psicoanálisis.

Este trabajo presenta una perspectiva histórica del concepto de inconsciente. Para ello se realiza un análisis de diversas fuentes documentales primarias que atañen al concepto de inconsciente y otras fuentes documentales de apoyo. En primer lugar, se desarrolla una breve perspectiva histórica del concepto, reconociendo que existen diversas teorías y formas de concebir al inconsciente, tanto en el terreno de la filosofía y la metafísica como en el ámbito de la psiquiatría y la psicología. En segundo lugar, se aborda la categoría de inconsciente en las teorizaciones realizadas por Freud. Por último, se justifica el sentido que Freud le otorga al inconsciente en el esquema de su teoría.

\section{Sobre el origen del término inconsciente}

Es una obviedad señalar que Freud no fue el primero en utilizar el término inconsciente (véase Brès, 2006; Brett, 1972; Charrier, 1970; Domínguez \& Yáñez, 2011; Ellenberger, 1976; Escobar-Maria, 2010; Landman, 1999; Mueller, 1976; Nicholls \& Liebscher, 2010; Tallis, 2002; Whyte, 1967). El término fue utilizado de diversas maneras y con distintas connotaciones por filósofos, psiquiatras, psicólogos, científicos, literatos y poetas a lo largo de la historia de la humanidad.

Rev. Latinoam. Psicopat. Fund., São Paulo, v. 15, n. 4, p. 891-907, dezembro 2012 
Hasta donde se tiene conocimiento, el término inconciente hizo su aparición en lengua inglesa en 1751 (Brès, 2006; Whyte, 1967) y recién en 1860 comienza a difundirse en Francia hasta que aparece formalmente en el Diccionario de la Academia Francesa en 1878 (Roudinesco \& Plon, 1998). En Rusia se tuvo noticias de un trabajo referido al inconsciente en 1875, aunque el tratamiento conceptual fuera más bien religioso y filosófico (Miller, 2005).

En Alemania también fue introducido muy tempranamente, aunque cobró mayor vigor durante el período romántico (Brès, 2006; Loureiro, 2005). A pesar de estos informes, que son muy anteriores a la emergencia del psicoanálisis, se puede reconocer que la concepción freudiana del inconsciente es heredera de una larga tradición filosófica y psiquiátrica que estuvo al alance de Freud y desde la cual pudo tomar algunas referencias (Bercherie, 1988; Assoun, 1991; Klappenbach, 1994).

Aun cuando el mismo Freud no establece una herencia filosófica explícita en su concepción teórica del psicoanálisis (Assoun, 1982, 1984; Tauber, 2009), es posible localizar en el término inconsciente diversas reminiscencias filosóficas. Pensadores como Arthur Schopenhauer y Eduard von Hartmann son algunas de los antecedentes que pueden citarse en la progenie filosófica. Sobre la influencia de las ideas de Schopenhauer en Freud (Barreira, 2009; Janaway, 2010; Young \& Brook, 1994) se han destinado más páginas que la recíproca de von Hartmann, aun cuando éste último fuera el primero en postular una "filosofía del inconsciente", ${ }^{1}$ titulo de su libro publicado en 1869 (Brès, 2006; Gardner, 2010; Jahoda, 1981).

No obstante, a pesar de que Freud pudiera conocer o no la obra de estos pensadores y aunque pudiera o no reconocerse deudor de sus postulados, fue a Theodor Lipps ${ }^{2}$ quien destacó como el precurso de la noción de inconsciente en el sentido más próximo al suyo (Assoun, 1982; Loparic, 2001). Lipps, aunque aparece como un ignoto para la mayoría de los psicoanalistas, fue una figura importante dentro de la psicología alemana de fines de siglo XIX (Kanzer, 1981; Loparic, 2001).

Lipps, que actuaba como profesor universitario, había ofrecido una conferencia en el III Congreso Internacional de Psicología, celebrado en Munich, en

${ }^{1}$ La Filosofía del inconsciente de von Hartmann tuvo una trascendental repercusión en el medio cultural y social alemán, de hecho, fue reeditada varias veces y también traducida a otros idiomas (Whyte, 1967).

${ }^{2}$ La influencia de Lipps sobre Freud puede rastrearse en dos direcciones, aunque divergentes, complementarias. Por una parte, en la noción de inconsciente y, por otra parte, en la concepción psicoanalítica del chiste.

Rev. Latinoam. Psicopat. Fund., São Paulo, v. 15, n. 4, p. 891-907, dezembro 2012 
1896, ${ }^{3}$ bajo el título: "El concepto de inconsciente en psicología" (Brès, 2006; Buchner, 1896). Se reconoce una alusión explícita de Freud a este trabajo de Lipps en un pasaje de la obra sobre los sueños (Brès, 2006; Loparic, 2001).

Quien también tomó noticias de aquella proclamación pública sobre la importancia del inconsciente como categoría central para la psicología fue un ruso, que estuvo muy bien informado del psicoanálisis y la psicología de su época (González, 2011). Vygotsky, en un texto que plantea toda una verdadera discusión metodológica de la psicología a principios de siglo XX, invoca la misma conferencia de Lipps (Vygotski, 1927).

Sin embargo, los intereses de Lipps estaban más centrados en la legitimación del inconsciente como una categoría de la psicología que en la presentación y justificación de una categoría vinculada con la vida mental de los seres humanos (Brès, 2006), al margen del gran reconocimiento que realiza del inconsciente como base general de la vida psíquica (véase Lipps, 1897).

En el campo de la psicología, varios autores también se aproximaron a la categoría de inconsciente, aunque bajo la concepción de que existen determinados fenómenos psíquicos que no llegan a ser procesados y reconocidos conscientemente. En este marco, Fechner, Herbart, Helmholtz y el propio Wundt fueron algunos de los autores que plantearon la existencia de ciertas ideas o percepciones que no alcanzaban el reconocimiento consciente porque no tenían la fuerza necesaria para superar el umbral requerido y por tanto no aparecían en el escenario de la consciencia (Araujo, 2012; Barbenza, 1990, 1997; Brett, 1972; Domínguez \& Yáñez, 2011; Honda, 2004).

Por su parte, en los anales de la primera psiquiatría dinámica también pueden vislumbrase antecedentes importantes en el camino de la conceptualización del inconsciente. Los fenómenos del sonambulismo, la personalidad múltiple y la histeria proporcionaron los primeros indicios de algo más allá de la consciencia (Bercherie, 1988; Ellenberger, 1976). En este marco, los primeros magnetizadores e hipnotizadores estuvieron muy cerca del reconocimiento del inconsciente, aunque desde luego, bajo una conceptualización y metodología muy diferente.

${ }^{3}$ Lipps fue el vicepresidente de ese tercer congreso (Piéron, 1954). Como dato, vale mencionar que fue en ese congreso donde se instituyó definitivamente la denominación de psicología como único calificativo convocante de la larga serie de congresos internacionales de psicología, dado que el primero llevó como título Congreso Internacional de Psicología Fisiológica, organizado en Paris en 1889 y el segundo fue denominado Congreso Internacional de Psicología Experimental, organizado en Londres en 1892 (véase Anónimo, 1976; Ardila, 1972; Montoro, Carpintero \& Tortosa, 1983; Piéron, 1954). A tono con cierto rigor histórico, el primigenio proyecto de organización de congresos internacionales de psicología se debe a Julian Ochorowicz, quien ya en 1881 había pensado en su organización (véase Nicolas \& Söderlund, 2005).

Rev. Latinoam. Psicopat. Fund., São Paulo, v. 15, n. 4, p. 891-907, dezembro 2012 
Más cercano en el tiempo a la elaboración freudiana del inconsciente, es conocida la disputa entre Freud y Janet respecto a la prioridad de algunas nociones, en particular, respecto al tratamiento analítico y la concepción de los fenómenos inconscientes (Anguera, 1991; Costa, 2008; Mannoni, 1997; Salas, 2010). De la misma manera que Freud y sus contemporáneos, Janet tuvo en sus manos el reconocimiento de algo más allá de la consciencia a través de la hipnosis. La opción que tomó Janet para nominar ese fenómeno alterado de consciencia fue la categoría de subconsciencia, aun cuando en su tesis de doctorado sobre el automatismo psicológico de 1889 hablara de "ideas fijas inconscientes" (Costa, 2008).

Como señala Rancière (2005), el inconsciente es uno de esos términos litigiosos, es decir, que convocan a la disputa intelectual por su reconocimiento y herencia. En ese terreno querellante, se plantea que la conceptualización freudiana del inconsciente tiene una deuda con la literatura, la estética y el arte del siglo XIX, donde su presencia ya estaba contenida (Görner, 2010; Rancière, 2005).

Al margen de estas controversias y los diversos avatares conceptuales del término, lo cierto es que con la categoría de inconsciente se ha tratado de señalar un aspecto del contenido mental que difiere de la consciencia, al cual el sujeto no tiene un acceso directo o accede a través de ciertas desfiguraciones. El inconsciente ha sido visto como el negativo de la consciencia. De hecho, algunos se han referido al inconsciente en términos de subconsciencia, destacándolo como un aspecto subordino al trabajo de la consciencia.

Por el calificativo inconsciente se pueden referir actos ejecutados sin consciencia, es decir, actos de inconsciencia como los actos irresponsables o peligrosos (Holanda, 2010). Lo inconsciente también puede representar lo más profundo de las pasiones humanas. Asimismo puede reflejar el costado de la sinrazón o la locura. Con lo inconsciente se sugiere la idea de una motivación oculta o significado latente. En fin, bajo el término inconsciente se pueden rastrear diversas filiaciones y concepción teóricas, así como diversas significaciones y connotaciones semánticas.

Será en el contexto del trabajo que inicia Freud en los umbrales del siglo XX que la noción de inconsciente adquiere un nuevo estatus y fundamento central para el funcionamiento del psiquismo humano. A pesar de los claros antecedentes de la noción de inconsciente que se han registrado, ni la filosofía, psiquiatría o psicología de la época llegaron a plantear una noción de inconsciente tal como aparece en el trabajo de Freud. Por tanto, sus teorizaciones son de crucial importancia para pesquisar el movimiento de legitimación de la noción de inconsciente en el psicoanálisis. 


\section{La historia del inconsciente psicoanalítico}

Sin dudas, la obra cumbre de Freud que dio paso a la introducción del psicoanálisis en la vida intelectual del pensamiento humano fue La interpretación de los sueños, obra publicada en 1899 , pero fechada en $1900 .{ }^{4}$ Esa obra es considerada como el punto de referencia que divide la producción anterior de Freud llamada pre-psicoanalítica y la nueva fase de producción propiamente psicoanalítica.

Recibe esta consideración por haber sido una obra en la que se propone un modelo del psiquismo inconsciente y se presenta un método de indagación de ese psiquismo. En ella se plantea que por medio de la interpretación de los sueños se confecciona una vía regia de acceso al inconsciente (Freud, 1900; Mannoni, 1997).

En la obra sobre los sueños, el inconsciente es conceptualizado como una instancia del "aparato psíquico", 5 cuyo rol es proporcionarle al sueño la fuerza constitutiva para su formación (Freud, 1900). En este trabajo, el deseo inconsciente es el punto de partida para la formación del sueño y el sueño es una formación psíquica que da cuenta de la existencia del inconsciente. El famoso capítulo siete del libro puede ser considerado como el texto fundacional, allí por primera vez se conceptualiza la noción de inconsciente y se describe su funcionamiento.

No obstante, aún antes de esta publicación, Freud ya había dado cuenta de la existencia de un fenómeno que se aproximaba al inconsciente, aunque todavía no lo formula como tal. Sin forzar demasiado los acontecimientos, se puede indicar que el período pre-1900 será un tiempo en el que Freud rondará la conceptualización del inconsciente. Esto acontece por medio de su contacto con la sugestión hipnótica ${ }^{6}$ y la práctica clínica en su consultorio particular.

${ }^{4}$ Como se sabe, Freud quiso que su manuscrito apareciera como una obra del siglo XX, por eso le encomendó al editor que le colocara la fecha de 1900, en lugar de la verdadera fecha de publicación que fue durante el mes de noviembre de 1899. Fue una obra que no tuvo mucha repercusión al comienzo, pero con el correr de los años sería ampliamente difundida y traducida a otros idiomas (Mannoni, 1997; Marinelli \& Mayer, 2011), como la primera al ruso en 1904 (Miller, 2005; Montalbán, 2011).

${ }^{5}$ Este "aparato psíquico", también llamado "aparato anímico" o "proceso mental”, es utilizado por Freud como una representación virtual, sin correspondencia con ninguna anatomía cerebral o condición del sistema nervioso. Se trata de un modelo que Freud elabora para representar las diversas instancias psíquicas y explicar sus características y funciones: inconsciente, preconsciente y consciencia.

${ }^{6}$ Para un examen breve sobre la historia de la hipnosis véase Chertok (1992) y Tortosa (1999).

Rev. Latinoam. Psicopat. Fund., São Paulo, v. 15, n. 4, p. 891-907, dezembro 2012 
En esos ámbitos, Freud pudo comprobar experimentalmente la existencia del inconsciente. Sin embargo, esta existencia no fue una comprobación estrictamente freudiana, más bien fue una comprobación que tuvo lugar en el marco de los trabajos sobre sugestión e hipnosis que el propio Freud había visto en la Salpêtrière con Charcot y en la Escuela de Nancy con Liébeault y Bernheim ${ }^{7}$ (Bercherie, 1988; Ellenberger, 1976; Fulgencio, 2002).

La hipnosis era un mecanismo por el cual se podían implantar ideas en el paciente o bien el recurso por el cual el médico podía indicar la realización de alguna acción, mientras los pacientes permanecían bajo hipnosis. Luego de volver del transe hipnótico, aquellos ejecutaban la consigna que el médico había establecido, aunque sin tener consciencia del acto y sin poder explicar la acción realizada. Por medio de este mecanismo, muy popularizado en la época, se manifestaba la división del psiquismo, entre fenómenos conscientes y fenómenos no conscientes.

Durante el período, pre-1900, Freud supo reconocer la existencia de un fenómeno que escapa por entero a la consciencia de los pacientes, pero no lo conceptualiza como inconsciente, ${ }^{8}$ sino de diversas formas. En la "Comunicación preliminar" de "Estudios sobre la histeria", obra publicada conjuntamente con Breuer, se hace referencia a diversos conceptos que suponen un registro que va más allá de la consciencia (Breuer \& Freud, 1895), pero todavía no se formula la noción de inconsciente (Baratto, 2009; Schutt, 1995). Como ejemplo, los conceptos utilizados para denominar el fenómeno inconsciente eran: doble consciencia, consciencia segunda, consciencia anormal, conciencia hipnoide o subconsciente.

La concepción de ciertos fenómenos que escapan al contenido de la consciencia era un dominio de la época. En ese contexto, la consciencia se ubicaba como la categoría rectora de lo mental y todo fenómeno que no fuera del orden de lo consciente se conceptualizaba como un subproducto. Es claro que

${ }^{7}$ Se sabe que entre la Escuela de la Salpêtrière y la Escuela de Nancy se suscitaron ásperas disputas con respecto a la forma de concebir la hipnosis (véase Anguera, 1991; Chertok, 1992; Tortosa, 1999).

${ }^{8}$ Chertok (1992) considera que el inconsciente y el desarrollo del psicoanálisis son el producto de las indagaciones sobre la hipnosis. Incluso argumenta que el método psicoanalítico se sostiene en la sugestión, a pesar de que Freud haya renunciado a la metodología de la hipnosis como forma de trabajo psicoterapéutico.

9“Sobre el mecanismo psíquico de los fenómenos histéricos. Comunicación preliminar” fue publicado en 1893, conjuntamente entre Freud y Breuer y luego incluido como primer capitulo en "Estudios sobre la histeria", que data de 1895 (Schutt, 1995).

Rev. Latinoam. Psicopat. Fund., São Paulo, v. 15, n. 4, p. 891-907, dezembro 2012 
estas primeras indagaciones sobre los estados de una segunda conciencia provenían del ámbito clínico y de las indagaciones de los fenómenos anormales de los pacientes de la época. Sin embargo, hasta ese momento, el inconsciente no aparecía conceptualizado sino bajo la forma de una consciencia de segundo orden, anormal o hipnoide.

Se puede argumentar, entonces, que Freud no llegó a conceptualizar el inconsciente en el período pre-1900 porque él mismo estaba dominado por el pensamiento y las ideas de la época, las cuales suscribían al dominio absoluto de la consciencia. Ese dominio de lo consciente no dejaba margen para conceptualizar otra noción que no sea un derivado de aquella. Puede resultar paradójico, pero lo cierto es que mientras en el ámbito filosófico, metafísico y cultural la categoría de inconsciente ya tenía un terreno ganado, en el ámbito clínico-psiquiátrico todavía no lograba su incorporación explícita como categoría mental. En ese terreno, el psicoanálisis freudiano proyecta su avanzada y la noción de inconsciente se vuelve una categoría operativa del funcionamiento mental.

Luego de la obra sobre los sueños, en la que se presenta el primer modelo del psiquismo ${ }^{10}$ y los conceptos de inconscientes, preconsciente y consciente, Freud se dedica a indagar los temas de la sexualidad humana y su compromiso en la causación de las neurosis, también se dedica a pulir la técnica psicoanalítica, escribe algunos casos ejemplificadores del tratamiento analítico y documenta algunos aspectos del inconsciente en la vida cotidiana.

En relación a esto último, Freud redactó una serie de textos en los que pudo certificar la motivación inconsciente de determinadas conductas de la vida social de las personas. ${ }^{11}$ El chiste, el lapsus, el olvido, los fallidos y el propio sueño son verdaderos actos psíquicos que ponen de manifiesto la viva expresión del contenido inconsciente. ${ }^{12}$ Durante estos trabajos, Freud no realizó una indagación muy profunda sobre la noción de inconsciente, no obstante, fue muy creativo a la hora de ejemplificar la expresión de lo inconsciente en el comportamiento y la vida cotidiana de las personas.

Será en el período de la construcción metapsicológica que Freud llegará a elaborar más profundamente sus concepciones teóricas acerca del inconsciente.

${ }^{10} \mathrm{El}$ segundo modelo del psiquismo fue presentado en el texto llamado "El yo y el ello" (Freud, 1923a).

${ }^{11}$ Para un examen de estos tópicos véanse los trabajos de Freud relacionados con "Psicopatología de la vida cotidiana" (Freud, 1901) y "El chiste y su relación con lo inconsciente" (Freud, 1905).

${ }^{12}$ Es interesante consignar como Freud utiliza la técnica de la interpretación de los sueños en el trabajo analítico, por ejemplo, en el famoso caso Dora (véase Iglesias, 2012).

Rev. Latinoam. Psicopat. Fund., São Paulo, v. 15, n. 4, p. 891-907, dezembro 2012 
La metapsicología ${ }^{13}$ fue el intento freudiano por elaborar una disciplina que diera cuenta del psiquismo humano, considerando una dinámica, una tópica y una economía del mismo. ${ }^{14} \mathrm{Si}$ bien este intento quedó inconcluso luego de varios trabajos ${ }^{15}$ no obstante, será un período muy fructífero de elaboración conceptual para el psicoanálisis.

Assoun (2002) sostiene que la metapsicología es un término que designa el límite entre lo especulativo y lo científico en la producción freudiana. En otras palabras, es una disciplina, un método y una especulación por medio de la cual se construyen los conceptos en psicoanálisis y fundamentalmente por medio del cual se aborda el conocimiento de los procesos inconscientes.

En el marco de la producción metapsicológica, Freud le dedicó un trabajo específico al tema del inconsciente. En su texto sobre "Lo inconsciente" (Freud, 1915), sostuvo varias cuestiones centrales. En primer lugar, postula al mecanismo de la represión como el censor que decide qué contenidos son del orden inconsciente y qué contenidos pueden llegar a ser del orden de lo consciente. Más aún, la represión es el concepto clave que da origen al psiquismo humano.

En segundo lugar, plantea que todo lo reprimido inexorablemente tiene que ser inconsciente, pero no necesariamente todo el contenido alojado en el inconsciente tiene que estar reprimido. En tercer lugar, sostiene que no hay un conocimiento directo del contenido de lo inconsciente sino a través de una versión deformada en la consciencia. En cuarto lugar, manifiesta que mediante la técnica del psicoanálisis se puede tener acceso al contenido inconsciente, aunque de una forma transfigurada.

${ }^{13}$ El término "metapsicología" apareció por primera vez en una carta personal dirigida Wilhelm Fliess, el 13 de febrero de 1896 y posteriormente mencionada en forma pública en 1904 en "Psicopatología de la vida cotidiana" (Assoun, 2002). Para Assoun (2002), "metapsicología" es un término que designa de otro modo al psicoanálisis.

${ }^{14}$ Conviene recordar que el funcionamiento del psiquismo freudiano es ubicado metapsicológicamente en tres registros: tópico (lugar o registro en el que se ubican los diferentes sistemas), económico (fuerza, energía, carga o investidura que contienen las representaciones) y dinámico (movimiento y conflicto entre las representaciones y sus investiduras) (Freud, 1915).

${ }^{15}$ Los trabajos incluidos en el contexto de la elaboración metapsicológica son: "Pulsión y destinos de pulsión" (1915), "Lo inconsciente" (1915), "La represión" (1915), "Duelo y melancolía" (1916) y "Complemento metapsicológico a la doctrina de los sueños" (1917). Por lo que se base, Freud había previsto la redacción de 12 trabajos metapsicológicos, entre los cuales se incluiría uno sobre la consciencia, no obstante, únicamente esos 5 trabajos fueron publicados. Luego de varios años se tuvo conocimiento de un ensayo inconcluso, que fue publicado bajo el título de Visión general de las neurosis de transferencia. Un ensayo metapsicológico, en 1986 (Assoun, 2002).

Rev. Latinoam. Psicopat. Fund., São Paulo, v. 15, n. 4, p. 891-907, dezembro 2012 
En este mismo trabajo, Freud describe el modo de funcionamiento y las características distintivas de los sistemas inconsciente, preconsciente y consciente. De una manera resumida, el inconsciente es caracterizado como el proceso primario y la consciencia es descripta como el proceso secundario, conjuntamente con el preconsciente (Gomes, 2003).

En un trabajo posterior, que muy bien puede inscribirse dentro de la producción metapsicológica, Freud vuelve sobre la noción de inconsciente, aunque con una nueva visión. En la obra "El yo y el ello" (Freud, 1923a), el inconsciente pasa a tener un lugar diferente en la estructuración de la vida mental. Con la introducción de las categorías de yo, ello y superyó, el inconsciente adquiere una nueva distribución topológica: su lugar de residencia es ello, aunque también mantiene ramificaciones en el yo y en el superyó.

Ahora el inconsciente ya no es sólo un sistema independiente y contrapuesto a los otros sistemas como en la primera tópica, sino que es parte de una estructura de funcionamiento más global. Con esta nueva versión del funcionamiento psíquico se pasa de una concepción intersistémica (primer tópica) a una intrasistémica (segunda tópica).

Aunque la segunda tópica suponga un nuevo entendimiento para el funcionamiento mental y, al mismo tiempo, represente una nueva legalidad para el inconsciente, todavía su carácter de concepto nuclear para el psicoanálisis mantiene su potencia. La noción de inconsciente seguirá siendo una de las categorías centrales del psicoanálisis, independientemente de las diversas connotaciones teóricas que fue adquiriendo con las diferentes corrientes psicoanalíticas que se fueron proyectando después de Freud.

\section{Conclusiones}

Es verdad que lo "inconciente" era examinado desde hacía mucho tiempo por filósofos como concepto teórico, pero aquí, en los fenómenos del hipnotismo, se volvió por vez primera algo vivo, palpable y objeto de experimentación.

(Freud, 1923b, p. 204).

En diversas oportunidades se ha discutido el carácter de originalidad y de descubrimiento del concepto de inconsciente por parte de Freud y el psicoanálisis. Al respecto, existe una vasta documentación que ha puesto de relieve que la noción de inconsciente reconoce, por lo menos, una doble herencia. Por una parte, la categoría de inconsciente venía siendo objeto de examen de diversos pensadores con una impronta filosófica y metafísica. Por otra parte, la psiquiatría ya

Rev. Latinoam. Psicopat. Fund., São Paulo, v. 15, n. 4, p. 891-907, dezembro 2012 
había documentado diversos fenómenos que ponían de manifiesto la existencia de algo más allá de la consciencia.

Dos obras pueden representar muy bien esa doble herencia: "Estudios sobre la histeria" y La interpretaciones de los sueños. En la primera, aunque sea una obra en coautoría y figure en el llamado período pre-psicoanalítico, se pone manifiesto diversas concepciones que prefiguran la noción de inconsciente. Desde ya, en esa obra se puede reconocer la herencia de la conceptualización psiquiátrica, aun cuando el trabajo posterior de Freud rompa con la práctica médica de la época. Por su parte, en la segunda se formula el concepto de inconsciente en el marco del psicoanálisis y se observa la herencia de la conceptualización filosófica.

Sería oportuno preguntarse a cuál de las dos tradiciones debe adscribirse la formulación de la categoría de inconsciente según el psicoanálisis iniciado por Freud. Para algunos autores se trata de una síntesis entre ambas tradiciones (Roudinesco \& Plon, 1998), mientras que otros autores se inclinan más por una u otra referencia como fundamento de la noción de inconciente.

Se reconoce que Freud tuvo una posición ambivalente con respecto a legitimación filosófica de sus ideas, más bien, su aspiración y pretensión siempre estuvo en el ideario científico, es decir, en la inscripción del psicoanálisis en el campo científico (Assoun, 1982; Gallegos, 2009; Tauber, 2009). Aun cuando esta inscripción sea discutible por el sistema de clasificación científica que se pregonaba en Alemania a fines de siglo XIX entre ciencia del espíritu y ciencia de la naturaleza (Assoun, 1991).

Bajo este trasfondo epistemológico y las diversas herencias teóricas, Freud confeccionó una nueva conceptualización del inconsciente en términos psicoanalíticos. Frente al dominio de las conceptualizaciones de la filosofía, la psicología y la psiquiatría, tuvo que producir diversas operaciones de legitimación conducentes a instalar la noción de inconsciente como categoría fundante del psiquismo y como noción central del psicoanálisis.

La primera operación de legitimación fue oponer la noción de inconsciente a todo el dominio de la filosofía y la psicología de la consciencia que imperaba como rectora del psiquismo en la época. De este modo, el inconsciente no sólo adquiría un lugar de supremacía en la vida mental, sino además, ya no podía se ser conceptualizado como un subproducto o el negativo de la consciencia.

La segunda operación de legitimación de lo inconsciente fue teorizarlo como un concepto implicado no solamente en la vida de los enfermos, sino además en la vida normal de los seres humanos. Por tanto, los sueños, los chistes, los lapsus y otros fenómenos de la cotidianeidad proporcionan las modalidades de pesquisajes del inconsciente en la vida normal de los seres humanos. 
La tercera operación de legitimación fue proporcionarle una estructura formal de funcionamiento, es decir, conceptualizar el inconsciente en la estructura y funcionamiento del aparato psíquico. Esta operación transcurre durante varios años, comenzando con la primera tópica, luego con las elaboraciones metapsicológicas y más tarde con la formulación de la segunda tópica del aparato psíquico.

En su conjunto, estas operaciones son el efecto de la avanzada del psicoanálisis como un nuevo saber sobre el psiquismo inconsciente, que la mentalidad de la época, a pesar de reconocer parcialmente su existencia, no supo o no quiso darle ese lugar nuclear que Freud le atribuyó en sus teorizaciones.

En ese contexto de legitimación, la obra sobre los sueños cumple con el cometido de postular una nueva conceptualización del funcionamiento psíquico, donde la noción de inconsciente también adquiere una nueva legalidad. De allí se desprende que la citada obra adquiera un valor simbólico fundacional para el psicoanálisis y que el propio Freud le atribuyera el carácter de "documento histórico" (Mannoni, 1997; Marinelli \& Mayer, 2011).

Aunque no pueda responderse taxativamente a nuestra pregunta sobre el lugar donde recae la herencia del inconsciente, se puede argumentar que tal herencia se ubica más del lado de la clínica psiquiátrica. Es en la clínica de los fenómenos histéricos donde se constata el sentido inconsciente de los síntomas. Desde luego, en los fenómenos de la vida cotidiana también se pueden inteligir las manifestaciones del inconsciente, pero es en la labor clínica donde el inconsciente adquiere su pleno sentido para el psicoanálisis.

En el terreno de la clínica, el inconsciente encuentra realmente su fundamento puesto que ya no se trata sólo del negativo de la consciencia como había sido conceptualizado en la tradición filosófica, más bien, se trata de un concepto que rige la vida anímica. Así el inconsciente es formalizado como un concepto tópico, económico y dinámico, lo que supone tanto un comercio como un conflicto entre los sistemas. Concluyentemente, en el ámbito de la clínica psicoanalítica el inconsciente tiene una operatividad muy diferente que en el ámbito de la filosofía.

\section{Referencias}

Anguera, B. (1991). Pierre Janet, un contemporáneo de Sigmund Freud. La noción de inconsciente. Anuario de Psicología, 50, 99-108.

Anónimo (1976). El XXI Congreso Internacional de Psicología (Paris). Revista Latinoamericana de Psicología, 8(3), 495.

Araujo, S. (2012). Why did Wundt abandon his early theory of the unconscious?

Rev. Latinoam. Psicopat. Fund., São Paulo, v. 15, n. 4, p. 891-907, dezembro 2012 
Towards a new interpretation of Wundt's psychological project. History of Psychology, 15(1), 33-49.

Ardila, R. (1972). La psicología contemporánea. Panorama internacional. Buenos Aires: Paidós.

Assoun, P-L. (1982). Freud. La filosofía y los filósofos. Barcelona: Paidós.

Assoun, P-L. (1984). Los fundamentos filosóficos del psicoanálisis. In R. Jaccard (comp.), Historia del psicoanálisis (Vol. 1). Buenos Aires: Juan Granica.

Assoun, P-L. (1991). Introducción a la epistemología freudiana. México: Siglo XXI.

Assoun, P-L. (2002). La metapsicología. México: Siglo XXI.

Baratto, G. (2009). A descoberta do inconsciente e o percurso histórico de sua elaboração. Psicologia: Ciência e Profissão, 29(1), 74-87.

Barbenza, C. (1990). Procesos inconscientes: un constructor necesario en los modelos psicológicos actuales. Boletín Argentino de Psicología, 3(4/5), 1-3.

Barbenza, C. (1997). Evolución de la problemática de los procesos inconscientes. Cuadernos Argentinos de Historia de la Psicología, 3(1/2), 35-54.

Barreira, I. (2009). Schopenhauer y Freud. Buenos Aires: Del Signo.

Bercherie, P. (1988). Génesis de los conceptos freudianos. Buenos Aires: Paidós.

Brès, I. (2006). El inconsciente. Buenos Aires: Autel.

Brett, G. (1972). Historia de la psicología. Buenos Aires: Paidós.

Breuer, J. \& Freud, S. (1987). Estudios sobre la histeria. In Sigmund Freud. Obras Completas (Vol. 2). Buenos Aires: Amorrortu. (Traballho original publicado em 1895).

Buchner, E. (1896). The Third International Congress of Psychology. Psychological Review, 3(6), 589-602.

Charrier, J. (1970). El inconsciente y el psicoanálisis. Buenos Aires: Proteo.

Chertok, L. (1992). Hipnosis y sugestión. México: Cruz.

Costa, M. (2008). Pierre Janet e os atos psíquicos inconscientes revelados pelo automatismo psíquico das histéricas. Revista Latinoamericana de Psicopatologia Fundamental, 11(2), 301-309.

Domínguez, A. \& Yánez, J. (2011). El inconsciente: una mirada sobre su historia y sus retos actuales. Psychologia Latina, 2(2), 172-183.

Ellenberger, H. (1976). El descubrimiento del inconsciente. Madrid: Gredos.

Escobar-Maria, C. (2010). El inconsciente freudiano y sus destinos. Tempo Psicoanalítico, 42(2), 403-424.

Freud, S. (1996). La interpretación de los sueños. In Sigmund Freud. Obras Completas (Vol. 5). Buenos Aires: Amorrortu. (Trabalho original publicado em 1900).

Freud, S. (2001). Psicopatología de la vida cotidiana. In Sigmund Freud. Obras Completas (Vol. 6). Buenos Aires: Amorrortu. (Trabalho original publicado em 1901).

Freud, S. (1991). El chiste y su relación con lo inconciente. In Sigmund Freud. Obras Completas (Vol. 8). Buenos Aires: Amorrortu. (Trabalho original publicado em 1905).

Rev. Latinoam. Psicopat. Fund., São Paulo, v. 15, n. 4, p. 891-907, dezembro 2012 
Freud, S. (1996). Lo inconsciente. In Sigmund Freud. Obras Completas (Vol. 14). Buenos Aires: Amorrortu. (Trabalho original publicado em 1915).

Freud, S. (1984). El yo y el ello. In Sigmund Freud. Obras Completas (Vol. 19). Buenos Aires: Amorrortu. (Trabalho original publicado em 1923a).

Freud, S. (1984). Breve informe sobre el psicoanálisis. In Sigmund Freud. Obras Completas (Vol. 19). Buenos Aires: Amorrortu. (Trabalho original publicado em 1923b).

Freud, S. (1980). Algunas lecciones elementales sobre psicoanálisis. In Sigmund Freud. Obras Completas (Vol. 23). Buenos Aires: Amorrortu. (Trabalho original publicado em 1938).

Fulgencio, L. (2002, junho). A compreensão freudiana da histeria como uma reformulação especulativa das psicopatologias. Revista Latinoamericana de Psicopatologia Fundamental, 11(2), 301-309.

Gardner, S. (2010). Eduard von Hartmann's Philosophy of the Unconscious. In A. Nicholls \& M. Liebscher (Eds.), Thinking the unconscious. Nineteenth-Century german thought (pp. 173-199). New York: Cambridge University Press.

Gallegos, M. (2009). La concepción científica del psicoanálisis y sus relaciones con otros campos disciplinares: aspectos históricos y epistemológicos. Memorias del I Congreso Internacional de Investigación y Práctica Profesional en Psicología, XVI Jornadas de Investigación y Quinto Encuentro de Investigadores en Psicología del Mercosur, 1, 508-512.

Gomes, G. (2003). A teoria freudiana da consciência. Psicologia: Teoria e Pesquisa, $19(2), 117-125$.

Gonzalez, A. (2011). Inconsciente: ¿un diálogo entre Freud y Vigotsky? Psychologia Latina, 2(2), 158-171.

Görner, R. (2010). The hidden agent of the self: towards an aesthetic theory of the nonconscious in German romanticism. In A. Nicholls \& M. Liebscher, M. (Eds.), Thinking the unconscious. Nineteenth-Century german thought (pp. 121-139). New York: Cambridge University Press.

Iglesias, I. (2012). Revisión crítica de la metodología freudiana: los "cambios de vía", un concepto olvidado. Psychologia Latina, 3(1), 37-44.

Holanda, A. (2010). Notas para uma reflexão sobre consciência e inconsciente na fenomenologia. Revista da Abordagem Gestáltica, 16(1), 45-53.

Honda, H. (2004). Notas sobre a noção de inconsciente em Wundt e Leibniz. Psicologia: Teoria e Pesquisa, 20(3), 275-277.

Jahoda, M. (1981). Freud y los dilemas de la psicología. México: Premia.

Janaway, C. (2010). The real essence of human beings: Schopenhauer and the unconscious will. In A. Nicholls \& M. Liebscher (Eds.), Thinking the unconscious. Nineteenth-Century german thought (pp. 140-155). New York: Cambridge University Press.

Rev. Latinoam. Psicopat. Fund., São Paulo, v. 15, n. 4, p. 891-907, dezembro 2012 
Kanzer, M. (1981). Freud, Theodor Lipps, and "Scientific Psychology". Psychoanalytic Quarterly, 50, 393-410.

Klappenbach, H. (1994). Diferentes problemas y tradiciones en la psicología del siglo XIX. Idea, 14, 63-80.

Landman, P. (1999). Freud. Madrid: Istmo.

Lipps, T. (2001). O conceito de inconsciente na psicologia. Natureza Humana, 3(2), 335356. (Trabalho original publicado em 1897).

Loparic, Z. (2001). Theodor Lipps: uma fonte esquecida do paradigma freudiano. $\mathrm{Na}$ tureza Humana, 3(2), 315-334.

Loureiro, I. (2005). Luzes e sombras. Freud e o advento da psicanálise. In A. Jacó-Vilela, A. Ferreira \& F. Portugal (comps.), História da psicologia: rumos e percursos. Rio de Janeiro: NAU.

Mannoni, O. (1997). Freud. El descubrimiento del inconsciente. Buenos Aires: Nueva Visión.

Marinelli, L. \& Mayer, A. (2011). Soñar con Freud. Buenos Aires: El Cuenco de Plata.

Miller, M. (2005). Freud y los bolcheviques. Buenos Aires: Nueva Visión.

Montalbán, F. (2011). Diálogo entre marxismo y psicoanálisis: vigencia del legado de V. N. Voloshinov. Universitas Psychologica, 10(1), 263-277.

Montoro, L., Carpintero, H. \& Tortosa, F. (1983). Los orígenes de los congresos internacionales de psicología. Revista de Historia de la Psicología, 4(1), 43-57.

Mueller, F. (1976). Historia de la psicología. Madrid: F.C.E.

Nicholls, A. \& Liebscher, M. (2010). Introduction: thinking the unconscious. In A. Nicholls \& M. Liebscher (Eds.), Thinking the unconscious. Nineteenth-Century german thought (pp. 1-25). New York: Cambridge University Press.

Nicolas, S. \& Söderlund, H. (2005). The project of an International Congress of Psychology by J. Ochorowicz (1881). International Journal of Psychology, 40(6), 395-406.

Pieron, H. (1954). Histoire succincte des congrès internationaux de psychologie. L'Année Psychologique, 54(2), 397-405.

Rancière, J. (2005). El inconsciente estético. Buenos Aires: Del Estante.

Roudinesco, E. \& Plon, M. (1998). Diccionario de psicoanálisis. Buenos Aires: Paidós. Salas, G. (2010). Ribot, Janet y Binet: pioneros de la psicología francesa. Eureka, 7(2), 11-22.

Schutt, S. (1995). El pensamiento freudiano en 1895: Estudios sobre la histeria cien años después. Anuario de Psicología, 67, 9-14.

Tallis, F. (2002). Hidden minds. A history of the unconscious. New York: Profile.

Tauber, T. (2009). Freud's philosophical path. From a science of mind to a philosophy of human being. Scandinavian Psychoanalytic Review, 32, 32-43.

Tortosa, F. (1999). La hipnosis. Una controversia interminable. Anales de Psicología, 15(1), 3-25.

Rev. Latinoam. Psicopat. Fund., São Paulo, v. 15, n. 4, p. 891-907, dezembro 2012 
Vygotski, L. (1991). El significado histórico de la crisis de la psicología. Una investigación metodológica. In L. S. Vygotski. Obras Escogidas (Vol. 1). Madrid: Visor/M.E.C. (Trabalho original publicado em 1927)

Young, C. \& Brook, A. (1994) Schopenhauer and Freud. International Journal of Psychoanalysis, 75, 101-118.

Whyte, L. (1967). El inconsciente antes de Freud. México: Joaquín Mortiz.

\section{Resumos}

(A noção de inconsciente em Freud: antecedentes históricos e elaborações teóricas)

Este trabalho aborda a noção de inconsciente em Freud a partir de uma perspectiva histórica. Esta noção é uma das categorias centrais da psicanálise e aparece em diferentes orientações teóricas. Em primeiro lugar, discutimos a origem da noção na reflexão filosófica e nas referências teóricas da psiquiatria. Em segundo, trabalhamos sobre a noção de inconsciente em construções teóricas de Freud. Finalmente, descrevemos as várias maneiras pelas quais Freud legitimou a noção de inconsciente no âmbito da psicanálise.

Palavras-chave: Inconsciente, Freud, história, psicanálise

(The notion of the unconscious in Freud: background and theoretical elaborations)

This work examines the notion of unconscious in a historical perspective. The unconscious is one of the central categories of psychoanalysis and recognizes various theoretical backgrounds. Firstly, the origin of the term in the philosophical reflection and in the theoretical references of psychiatry is exposed. Secondly, the unconscious in the theoretical elaborations of Freud is developed. Finally, the different ways used by Freud to legitimate the unconscious mind in the psychoanalytical frame are exposed.

Key words: Unconscious, Freud, history, psychoanalysis

(La notion d'inconscient dans Freud: des antécédents et de élaborations théorique)

Cet article aborde la notion de l'inconscient depuis une perspective historique. La notion de l'inconscient est l'une des catégories centrales de la psychanalyse et reconnaît divers antécédents théoriques. En premier lieu, s'expose l'origine de la notion dans la réflexion philosophique et dans les références théoriques de la psychiatrie. En deuxième lieu, se travail la notion de l'inconscient dans les élaborations théoriques de Freud. Finalement, se décrit les diverses manières dont Freud légitiment la notion de l'inconscient dans le cadre de la psychanalyse.

Mots clés: Inconscient, Freud, histoire, psychanalyse 
(Die Frage der Grundlage in Freud: Elemente der angewandten Psychoanalyse)

Unter den vielen Reflexionen, welche die Diskussion um den Einschluss der Psychoanalyse und der Psychoanalytiker in den Bereich der geistigen Gesundheit hervorrufen, wird hier der Orientierungswert von dem, was Freud unter grundlegende Fragen im Bereich der Psychoanalyse versteht, in Frage gestellt. Dazu werden die Textstellen betrachtet, in denen der Autor diese Frage diskutiert; sei es im Rahmen der metapsychologischen Schriften, der technischen Darlegungen oder der Textstellen, in denen er Überlegungen über die Möglichkeiten der in der Therapie angewandten Psychoanalyse anstellt.

Schlüsselwörter: Freud, angewandte Psychoanalyse, Grundlage, Therapeutik

Citação/Citation: Gallegos, M. La noción de inconsciente en Freud: antecedentes históricos y elaboraciones teóricas (2012, dezembro). Revista Latinoamericana de Psicopatologia Fundamental, 15(4), 891-907.

Editor do artigo/Editor: Prof. Dr. Paulo Dalgalarrondo e Profa. Dra. Ana Maria G. R. Oda

Recebido/Received: 13.3.2012 / 3.13.2012 Aceito/Accepted: 25.5.2012 / 5.25.2012

Copyright: (C) 2009 Associação Universitária de Pesquisa em Psicopatologia Fundamental/ University Association for Research in Fundamental Psychopathology. Este é um artigo de livre acesso, que permite uso irrestrito, distribuição e reprodução em qualquer meio, desde que o autor e a fonte sejam citados / This is an open-access article, which permits unrestricted use, distribution, and reproduction in any medium, provided the original author and source are credited.

Financiamento/Funding: $\mathrm{O}$ autor declara ter sido financiado pelo Consejo Nacional de Investigacio0nes Científicas y Técnicas (Conicet), Argentina / / This research was funded by the Consejo Nacional de Investigacio0nes Científicas y Técnicas (Conicet), Argentina

Conflito de interesses/Conflict of interest: $\mathrm{O}$ autor declara que não há conflito de interesses / The author declares that has no conflict of interest.

\section{Miguel Gallegos}

Psicólogo, docente e investigador de la Facultad de Psicología de la Universidad Nacional de Rosario - UNR-Conicet (Rosário, Santa Fé, Ar).

Irice-Conicet

Bv 27 de Febrero 210 Bis

20000 Rosario, Santa Fé, Ar

e-mail: maypsy@yahoo.com.ar

Rev. Latinoam. Psicopat. Fund., São Paulo, v. 15, n. 4, p. 891-907, dezembro 2012 